\title{
Sympathetic Inhibition of Accommodation after Sustained Nearwork in Subjects with Myopia and Emmetropia
}

\author{
Balamurali Vasudevan, ${ }^{1}$ Kenneth J. Ciuffreda,${ }^{1}$ and Bernard Gilmartin ${ }^{2}$
}

Punpose. The purposes of the present study were to assess the effect of a sympathetic inhibitory pharmacologic agent, timolol maleate, on the magnitude of nearwork-induced transient myopia (NITM) and its decay in different refractive groups for an extended near task duration and to determine the proportion of the young adult population manifesting effective sympathetic access under naturalistic closed-loop viewing conditions.

Methods. Ten subjects with emmetropia and 10 with myopia were tested. They read binocularly for 1 hour at a distance of 35 to $40 \mathrm{~cm}$. NITM was calculated as the difference in distance refractive state after task as compared with before task immediately after reading. All subjects received timolol maleate to block the sympathetic nervous system and betaxolol as a control agent in independent test sessions separated by at least 3 days. Forty minutes after drug instillation, the NITM measurement procedure was repeated.

RESULTs. Initial NITM magnitude was larger in subjects with myopia than in subjects with emmetropia before and after timolol instillation. Furthermore, NITM magnitude in subjects with sympathetic access was increased after timolol instillation. In contrast, with the control agent betaxolol, there was no increase. NITM decay duration to baseline was increased after timolol instillation in the subjects with myopia only. Only $15 \%$ of the subjects ( $n=3$ subjects with myopia) demonstrated effective and significant access to sympathetic facility.

Conclusions. Subjects with myopia demonstrated an increase in decay duration with timolol, thus suggesting impaired sympathetic inhibition of accommodation. This may be a precursor for myopia progression in some persons. (Invest Ophthalmol Vis Sci. 2009;50:114-120) DOI:10.1167/iovs.08-1762

A ccommodation refers to the process whereby alterations occur in the dioptric power of the crystalline lens to obtain and maintain a clear image of an object at the fovea when focus is changed. ${ }^{1}$ It is produced by systematic variations in the lenticular and extralenticular structures of the human eye. ${ }^{1}$ The accommodative system receives dual pharmacologic innervation from the sympathetic and parasympathetic divisions of the autonomic nervous system. ${ }^{2}$

From the ${ }^{1}$ State University of New York, State College of Optometry, New York, New York; and ${ }^{2}$ Ophthalmic Research Group, School of Life and Health Sciences, Aston University, Birmingham, United Kingdom.

Submitted for publication January 18, 2008; revised May 15 and June 24, 2008; accepted November 3, 2008.

Disclosure: B. Vasudevan, None; K.J. Ciuffreda, None; B. Gilmartin, None

The publication costs of this article were defrayed in part by page charge payment. This article must therefore be marked "advertisement" in accordance with 18 U.S.C. $\$ 1734$ solely to indicate this fact

Corresponding author: Balamurali Vasudevan, State University of New York, State College of Optometry, 33 West 42nd Street, New York, NY 10036; bvasudevan@sunyopt.edu.
The sympathetic and parasympathetic systems have complementary actions. Parasympathetic input is mediated by the action of acetylcholine on muscarinic receptors, whose level of excitatory stimulation results in rapid ( $\sim 1$-second) changes in the accommodative state. ${ }^{2,3}$ Although several types of muscarinic (cholinergic) receptors have been found $\left(M_{1}, M_{2}, M_{3}\right.$, $M_{4}$ ), the $M_{3}$ receptor is primarily involved in ciliary muscle contraction. In contrast, the sympathetic receptors in the ciliary smooth muscle are principally inhibitory $\beta 2$ receptors and possibly inhibitory $\alpha$ receptors., ${ }^{2,3}$ The sympathetic system is inhibitory in nature and thus acts to produce a relative decrease in accommodation. ${ }^{4}$ Furthermore, the sympathetic system is slow in onset ( $\sim 40$ seconds) and smaller in effect than the parasympathetic system. ${ }^{4,5}$

The ciliary muscle is one of the most important extralenticular components of the eye involved in accommodation because its force deforms the crystalline lens ${ }^{1}$ to produce clear retinal imagery. It receives sympathetic stimulation primarily from $\beta 2$ adrenergic receptors. ${ }^{2}$ Approximately $90 \%$ of the $\beta$-receptors are of the type 2 variety. ${ }^{6}$ Gilmartin and Hogan ${ }^{7}$ found that with instillation of a $\beta$ agonist, tonic accommodation decreased $0.50 \mathrm{D}$ to produce a hyperopic shift, whereas with instillation of a $\beta$-blocker, it increased $1.00 \mathrm{D}$ to produce a myopic shift. $\beta$-Blockers may also influence the closed-loop far point of accommodation and open-loop accommodative adaptation. ${ }^{8}$ Hence, to understand better the mechanism of accommodation and its possible relation to myopia, use of a pharmaceutical agent that blocks the sympathetic system gains significance.

Two models have been proposed that attempt to link sympathetic inhibition of accommodation and myopia progression. ${ }^{9}$ The first model assumes a deficit in sympathetic input alone, in association with normal parasympathetic input. This would result in enhanced accommodative aftereffects, such as nearwork-induced transient myopia (NITM). ${ }^{9,10}$ In addition, it would produce a relatively prolonged period of decay of any induced myopic shift after nearwork. The second model assumes a deficit in the sympathetic and parasympathetic inputs. That is, because the level of sympathetic activation is positively correlated with the level of parasympathetic activity, a decrease in the latter would lead to a decrease in the former, thus producing a decrease in the accommodative response during nearwork followed by an enhanced accommodative aftereffect during subsequent far viewing. ${ }^{11}$ All of these effects are potentially myopiagenic. ${ }^{9-11}$

Several studies have reported on sympathetic inhibition related to decay of the accommodative response under openloop viewing conditions in the dark (for a review, see Chen et al. ${ }^{\text {?) }}$. These studies have primarily measured accommodative adaptation after short durations of nearwork and then reassessed the same after instillation of a pharmaceutical agent such as timolol (a nonselective $\beta$-antagonist) or betaxolol (a $\beta 1$-selective antagonist). Whereas timolol produces a decrease in intraocular pressure and an increase in tonic accommodation, a pharmacologic control agent such as betaxolol produces a decrease in intraocular pressure only ${ }^{12}$ and thus serves 
as a selective control agent. For example, Gilmartin and Bullimore ${ }^{13}$ measured accommodative adaptation for visual tasks at $0.3 \mathrm{D}$ and $5 \mathrm{D}$ in young adults with emmetropia. For the 5-D task, they reported that the post-task accommodative response decayed to baseline within 60 seconds, whereas with the addition of topical instillation of timolol, the decay duration increased to 80 seconds. However, this effect was not observed at the lower accommodative stimulus level, which typically decayed in less than 50 seconds for both conditions. Presumably this was because of the relatively low pharmacologic drive from the parasympathetic system. ${ }^{2}$ Hence, when sympathetic innervation was blocked and considerable accommodation was activated, decay duration was increased. Later, Gilmartin and Bullimore ${ }^{14}$ measured accommodative adaptation in subjects with late-onset myopia (LOM) and in subjects with emmetropia after a 10-minute visual counting task at distances equivalent to 1,3 , and $5 \mathrm{D}$. They reported that the decay duration of accommodative adaptation after nearwork was significantly increased in subjects with myopia as compared to subjects with emmetropia at the higher accommodative levels only (3 and 5 D). Again, lack of effect was found for the lowest stimulus level. From this and other findings, Gilmartin and Winfield ${ }^{12}$ assumed that the role of sympathetic innervation of the ciliary muscle may be, for example, to attenuate the accommodative response induced by periods of intense close work and thus reduce the risk of larger and prolonged post-task transitory pseudomyopic changes. Based on the findings of previous investigations, ${ }^{14,15}$ Gilmartin and Bullimore ${ }^{14}$ proposed that the onset of LOM (e.g., because of myopic nearwork susceptibility ${ }^{1,16,17}$ ) might follow a progressive sequence: a sympathetic inhibitory deficit according to model one described earlier, followed by a propensity to exhibit an accommodative aftereffect after nearwork, and the resultant retinal defocus/blur that would be cumulative because of this adaptive process. This would result in increased vitreal chamber depth and, hence, axial myopia. Later, Gilmartin and Winfield ${ }^{12}$ measured the open-loop accommodative decay after a 3-minute near task with the pharmacologic addition of timolol or betaxolol. They reported similarity in accommodative response profiles to the $\beta$-receptor antagonists in the subjects with early-onset myopia (EOM), LOM, and emmetropia. Thus, the deficit in sympathetic inhibition appeared to be independent of refractive state.

There is a clear association between sustained near vision and the development of myopia (for reviews, see Rosenfield and Gilmartin ${ }^{18}$ ). Thus, sympathetic inhibition may have an important etiological role in the development of certain classes of myopia in predisposed persons. ${ }^{5,8,15}$ However, open-loop accommodative responses are not representative of real-life situations in which blur-related visual feedback is present. Hence, knowledge of the analogous closed-loop accommodative response properties (NITM) gains importance. ${ }^{11}$

The closed-loop distance accommodative response after sustained nearwork, namely NITM, has been assessed before and after blockade of the sympathetic system in earlier studies. For example, Winn et al. ${ }^{19}$ reported that when timolol was used in emmetropic subjects, both the gain of the dynamic accommodative response for a target of low temporal frequency $(\sim 0.3 \mathrm{~Hz}$ or less) and the near-to-far closed-loop stepwise accommodative response increased. Thus, an increase in NITM decay time may be predicted under these conditions. Later, Mallen et al. ${ }^{20}$ measured open- and closed-loop post-task accommodative responses in subjects with emmetropia and subjects with myopia (LOM and EOM) after a near task of either 10 seconds or 3 minutes. In subjects with effective access to sympathetic facility, instillation of timolol resulted in an increase in the accommodative decay to baseline for the 3-minute task. However, no difference was observed for the 10-second task because this duration was too short for any substantial drug effect to occur given the slow temporal characteristics of sympathetic inhibition. In contrast, betaxolol produced no difference in time constant for either the 10-second or 3-minute task period. The Mallen et al. ${ }^{20}$ results suggested that sympathetic inhibitory facility was effective in only a proportion of the subjects (27\% of the subjects with emmetropia, $21 \%$ of the subjects with EOM, and $29 \%$ of the subjects with LOM). Not all subjects exhibited this feature, and the frequency distribution was relatively similar in each group. Thus, it appears that a significant proportion of the population may have a reduced propensity to myopia attributable to the presence of a sympathetic inhibitory facility that is independent of refractive status. However, these findings were never confirmed for longer durations of nearwork (e.g., 1 hour) in young adults.

The aims of the present investigation were threefold: first, to assess the magnitude of NITM after a 1-hour near task, with and without blockade of the sympathetic system; second, to ascertain refractive error dependency of the sympathetic inhibitory function; and third, to determine the percentage of the population with effective and significant access to the latter.

\section{MeTHODS}

\section{Subjects}

Twenty optometry and graduate students (10 subjects with emmetropia and 10 subjects with myopia) were recruited from the SUNY State College of Optometry. They ranged in age from 21 to 35 years (mean age, 24). There were 9 men and 11 women. All had normal vision function. They constituted a subgroup of subjects from an earlier related study in our laboratory. ${ }^{10}$ The noncycloplegic refractive status of each subject was obtained with an objective, open-field, infrared autorefractor (R-1; Canon, Lake Success, NY). This information was used to classify the subjects as those with myopia or those with emmetropia. It was also confirmed subjectively. Subjects with myopia had a spherical equivalent refraction that ranged from -0.5 to $-6 \mathrm{D}$, with a mean of $-2.12 \mathrm{D}$. Subjects with emmetropia had a spherical equivalent refraction that ranged from $+0.5 \mathrm{D}$ to $-0.25 \mathrm{D}$, with a mean of $+0.15 \mathrm{D}$. The cylindrical component was $\leq-1.00 \mathrm{D}$, with a mean of -0.64 D. All subjects with myopia were corrected with their habitual soft contact lenses, which resulted in a distance visual acuity of 20/20 or better monocularly and binocularly at distance and near. Subjects with a history of respiratory disorders, glaucoma, or narrow anterior chamber angles were excluded from the study. Informed consent was obtained from each subject after the nature and possible consequences of the study were explained. The research followed the tenets of the Declaration of Helsinki and was approved by the college's internal review board.

\section{Instrumentation}

All measurements of refractive state were obtained objectively (R-1; Canon), which is a widely used, binocular, open-field, infrared autorefractor for vision research. ${ }^{5}$ This instrument provides rapid measurements (approximately every 2 seconds) of refractive state. The power range is $\pm 15 \mathrm{DS}$ and $-7 \mathrm{DC}$, the dioptric resolution is $0.12 \mathrm{D}$, and the cylindrical axis resolution is $1^{\circ}$ (for a detailed explanation, see McBrien and Millodot ${ }^{21}$ ).

\section{Procedures}

Figure 1 shows a schematic representation of the methodology. Closed-loop accommodative response measurements are described below.

Pre-task. All subjects were seated in total darkness for 5 minutes to allow for the dissipation of any transient accommodative effects. ${ }^{22}$ The distance refractive state was measured in the right eye, while the 

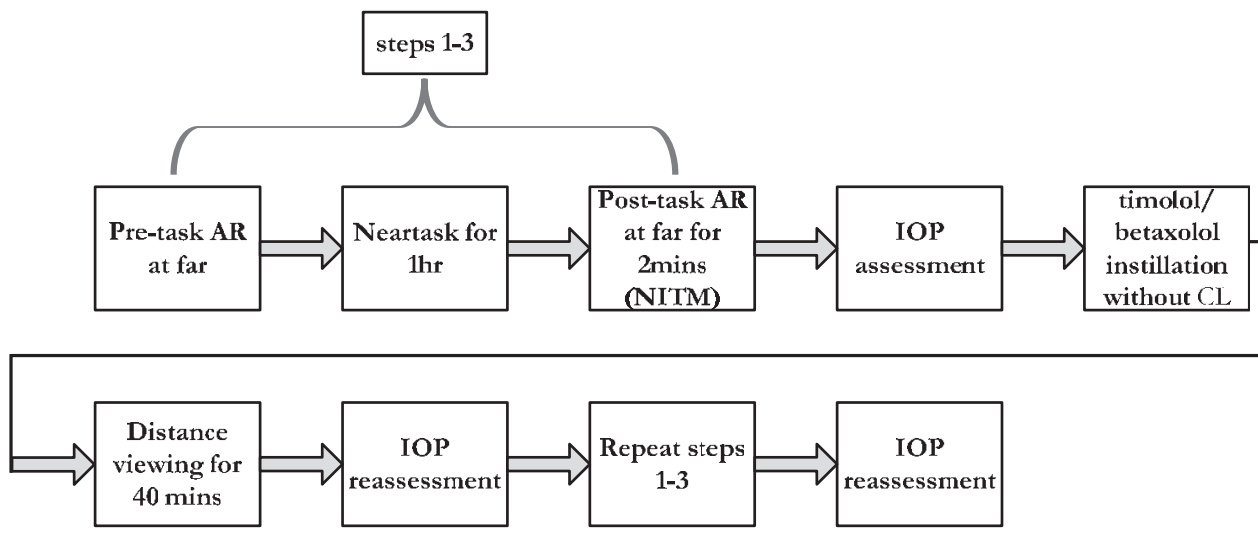

Figure 1. Flow chart of the experimental methodology. CL, contact lens.

subject binocularly viewed 20/30 Snellen letters at $6 \mathrm{~m}$. During all test periods, contact lenses were worn by the subjects with myopia to correct their distance refractive state and to avoid the potential of spectacle reflections that might interfere with the measurements. ${ }^{23}$ Twenty measurements of the distance refractive state were obtained with the autorefractor (R-1; Canon) with the subjects gazing at the distant target, and the spherical equivalent was calculated to the corneal plane.

Task. Subjects were seated at a desk in the laboratory under fluorescent room illumination and read optometric lecture notes at a distance of 35 to $40 \mathrm{~cm}$ during the 1-hour test period. They were instructed to maintain the text in focus at all times, and the distance of the reading material was assessed every 15 minutes by the examiner.

Post-task. Immediately after the 1-hour period of reading, subjects were quickly ( $\sim 2$ seconds) seated in the autorefractor and asked to focus on the distant Snellen target, and the distance refractive state was assessed every 2 seconds for a period of 120 seconds. Subjects were questioned about target clarity periodically to ensure that they were focusing accurately.

\section{Pharmaceutical Agents}

Two pharmaceutical agents, timolol maleate $(0.5 \%$; Alcon, Fort Worth, $\mathrm{TX})$ and betaxolol $\mathrm{HCl}(0.5 \%$; Alcon), were used to block the sympathetic adrenoceptors without change in pupil diameter. ${ }^{2}$ Timolol acts on the ciliary smooth muscle to modulate autonomic control of the accommodative response and to decrease intraocular pressure (IOP). ${ }^{12}$ In contrast, betaxolol was used as a pharmaceutical control; it only produces a decrease in IOP and thus differentiates the interaction between the changes in IOP and accommodation. Before instillation of these drugs, one drop of $0.5 \%$ proparacaine $\mathrm{HCl}$ was administered in the each eye to inhibit reflex tearing on the subsequent instillation of the $\beta$ antagonist drug.

After completion of the closed-loop accommodative measurements, the contact lenses were removed, and the IOP was measured in each eye with a noncontact pneumatic tonometer (T1; Canon, Tokyo, Japan). Then one drop of timolol (0.5\%) was instilled in each eye. Subjects were instructed to reinsert their contact lenses 10 minutes after instillation of the timolol and to gaze on the distant target at all times. Forty minutes after the timolol was instilled, IOP was measured again, and the entire process was repeated immediately after completion of the 1 hour of nearwork.

\section{Control Study}

All subjects served as their own controls. They were randomly allocated either timolol or betaxolol at an interval of at least 3 days. The same test paradigm described earlier was used to measure the closedloop accommodative response before and after betaxolol instillation. To achieve an ocular hypotensive effect approximately equivalent to that of timolol, ${ }^{12}$ two drops of betaxolol (0.5\%) were administered 5 minutes apart in each eye. IOP was measured before and after the task.
When compared with the timolol findings, these results reveal the effect of the sympathetic inhibitory system on the accommodative response characteristics under closed-loop viewing conditions.

\section{Data Analysis}

The data for each subject were divided into 10-second bin intervals. Each bin contained five data points that were averaged within and across subjects within each refractive group. ${ }^{11}$ Initial NITM was calculated from the five readings within the first 10 -second bin. The posttask minus pre-task difference in distance refractive state represented the NITM dioptric magnitude. The time taken for the NITM magnitude to dissipate in each subject, i.e., the time to decay to the pre-task distance baseline level, was calculated for each subject, and then it was averaged within each refractive group to obtain the mean decay duration. Regression analysis was conducted to determine mathematically the decay duration. These calculations of the initial NITM magnitude and its decay were obtained with and without use of the pharmaceutical agents.

The difference in initial NITM magnitude and decay duration obtained before and after timolol administration was calculated. Subjects with a positive difference in initial NITM magnitude, and above the noise level of the Canon autorefractor $\left(0.06 \mathrm{D}^{11}\right)$, were identified and classified as having access to sympathetic inhibition during the near task. These subjects also exhibited longer (minimum 10 seconds) decay duration after timolol instillation. Subjects with either no difference or a negative difference were classified as not having sympathetic access.

\section{Results}

\section{Initial NITM Magnitude}

Refractive Error. The initial NITM magnitude was assessed immediately after 1 hour of nearwork before and after the instillation of timolol (Fig. 2). Group means were the same $(0.22 \mathrm{D})$ before and after the instillation of timolol, as shown in Figure 2. The myopic subgroup manifested means of $0.28 \mathrm{D}$ and $0.27 \mathrm{D}$, respectively; the emmetropic subgroup manifested means of $0.14 \mathrm{D}$ and $0.16 \mathrm{D}$, respectively. Two-way, repeatedmeasures ANOVA for the factors of refractive error and drug effect was performed with respect to the initial NITM magnitude. It revealed a significant effect for refractive group $\left(F_{(1,32)}\right.$ $=12.39 ; P=0.001)$ but not for drug effect $\left(F_{(1,32)}=0.004\right.$; $P=0.94)$. Interaction effects were not significant $(P>0.05)$. A post hoc Fisher LSD test revealed that the initial NITM was larger in subjects with myopia than in subjects with emmetropia both before $(P<0.01)$ and after $(P=0.03)$ timolol instillation.

Sympathetic Access. Subjects were assessed with respect to their ability to demonstrate effective sympathetic access 


\section{Initial NITM}

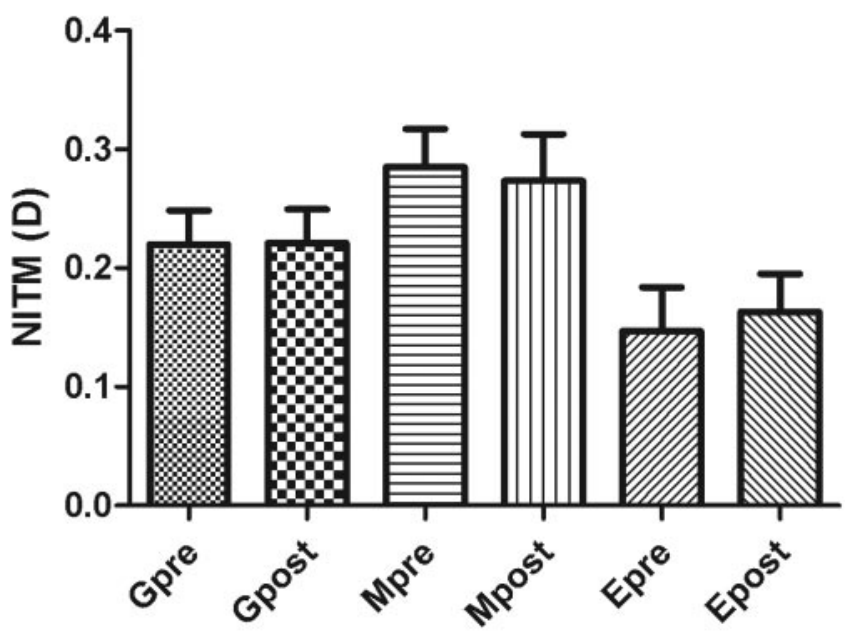

Figure 2. Mean initial NITM plotted as a function of group and refractive subgroup after 1 hour of nearwork before and after instillation of timolol. Plotted is the mean + 1 SEM. G, group; M, subjects with myopia; E, subjects with emmetropia.

based on our specified criterion (Fig. 3). In subjects with access to sympathetic facility, the initial NITM means before and after timolol instillation were $0.15 \mathrm{D}$ and $0.28 \mathrm{D}$, respectively. In subjects without sympathetic access, the NITM means before and after timolol instillation were $0.25 \mathrm{D}$ and $0.19 \mathrm{D}$, respectively. Two-way, repeated-measures ANOVA for the factors of sympathetic access and drug effect was performed. No effect was observed with respect to sympathetic $\operatorname{access}\left(F_{(1,32)}=0.001 ; P=0.96\right)$ or drug $\operatorname{effect}\left(F_{(1,32)}=0.78\right.$; $P=0.38)$. Interaction effects were significant $(P=0.03)$. A post hoc Fisher-LSD test revealed a significant effect $(P=0.05)$ for increased initial NITM magnitude in subjects with effective sympathetic access after timolol application. Other relevant comparisons were not significant.

\section{Initial NITM}

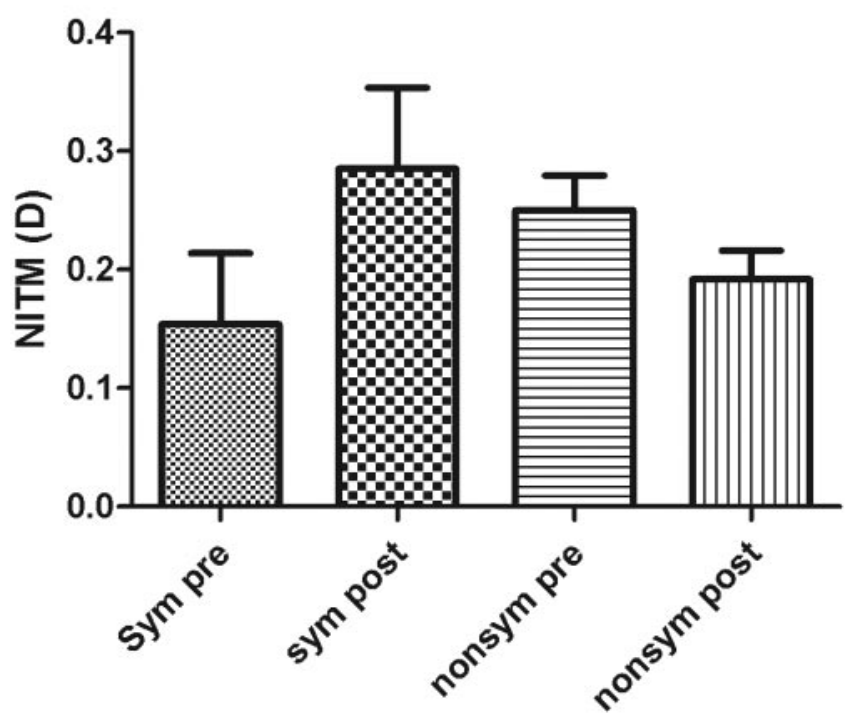

FIGURE 3. Mean initial NITM plotted after 1 hour of nearwork before and after instillation of timolol. Plotted is the mean +1 SEM. sym, sympathetic access; non-sym, without sympathetic access.

\section{Initial NITM}

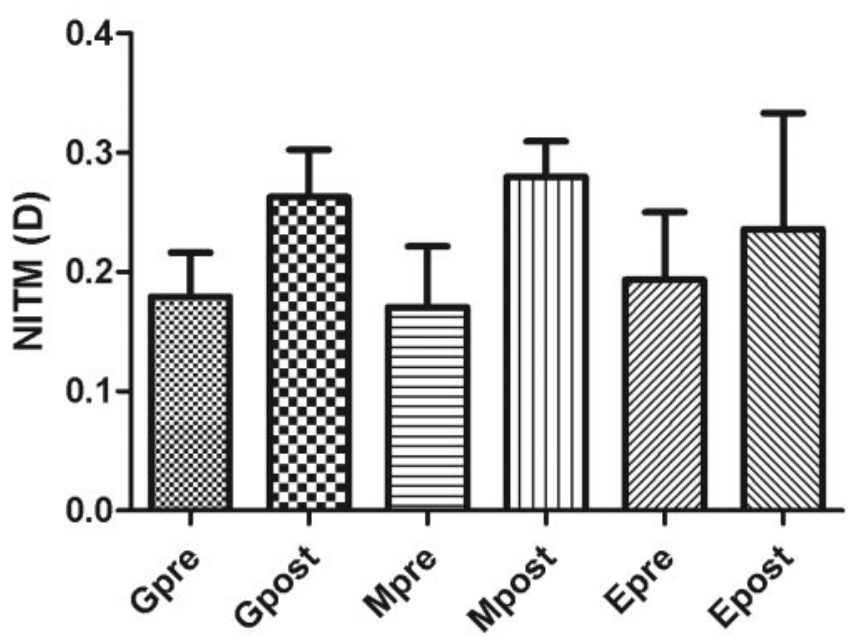

Figure 4. Mean initial NITM plotted as a function of group and refractive subgroup after 1 hour of nearwork before and after instillation of betaxolol. Plotted is the mean +1 SEM.

Of the seven subjects exhibiting results suggesting effective sympathetic access, four were emmetropic and three were myopic. Initial NITM means before and after timolol instillation were $0.22 \mathrm{D}$ and $0.39 \mathrm{D}$ in subjects with myopia and $0.08 \mathrm{D}$ and $0.15 \mathrm{D}$ in subjects with emmetropia, respectively. Paired $t$-tests were performed to compare the initial NITM magnitude within each of these refractive groups. Only the subjects with myopia exhibited a significant increase in NITM with timolol $\left(t_{(2)}=-22.51 ; P<0.01\right)$; the subjects with emmetropia did $\operatorname{not}\left(t_{(3)}=-2.54 ; P=0.08\right)$.

Control. Initial NITM magnitude was assessed after 1 hour of nearwork before and after instillation of betaxolol (Fig. 4). The group means NITM were $0.17 \mathrm{D}$ and $0.26 \mathrm{D}$, respectively. The myopic subgroup manifested NITM magnitude means of $0.17 \mathrm{D}$ and $0.27 \mathrm{D}$, respectively; the emmetropic subgroup exhibited NITM magnitude means of $0.19 \mathrm{D}$ and $0.23 \mathrm{D}$, respectively. Two-way, repeated-measures ANOVA for the factors of refractive error and drug effect was performed. It was not significant for either drug effect $\left(F_{(1,16)}=1.73 ; P=0.20\right)$ or refractive group $\left(F_{(1,16)}=0.03 ; P=0.85\right)$. Interaction effects were not significant $(P>0.05)$

\section{Decay of NITM}

Refractive Error. NITM decay was assessed as the time to reach the zero pre-task baseline (Fig. 5). Group means decay to baseline before and after timolol instillation were 50.7 seconds and 66.8 seconds, respectively. In the subjects with emmetropia, the decay to baseline means before and after timolol instillation were 47.2 and 49.4 seconds, respectively. In the subjects with myopia, the decay to baseline means before and after timolol instillation were 54 and 82.5 seconds, respectively. Two-way, repeated measures ANOVA for the factors of drug effect (timolol) and refractive group was performed. It revealed significance for the drug effect $\left(F_{(1,44)}=4.02 ; P=\right.$ $0.05)$ but not for the refractive group $\left(F_{(1,44)}=2.39 ; P=0.13\right)$. Interaction effects were not significant $(P>0.05)$. A post hoc Fisher-LSD test revealed that decay duration after the instillation of timolol was increased in the subjects with myopia compared to the subjects with emmetropia $(P=0.02)$. The mean decay duration for the subjects with myopia was increased after timolol instillation as compared with their preinstillation value $(P=0.04)$. 


\section{Refractive group-Timolol}

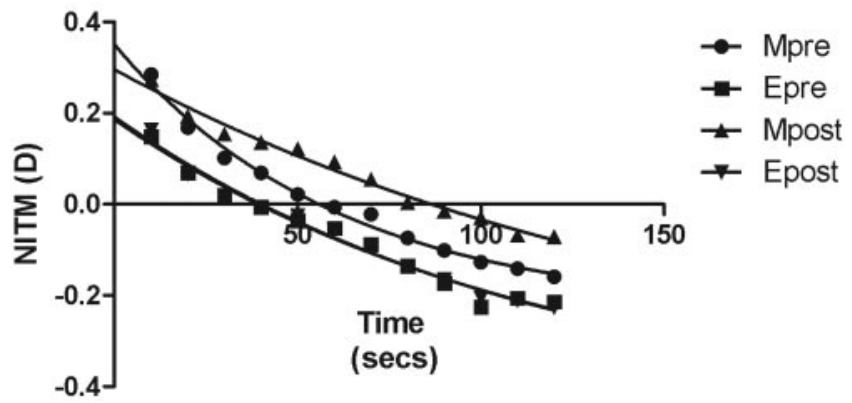

FIGURE 5. Decay of NITM as a function of refractive subgroup after 1 hour of nearwork before and after instillation of timolol. Plotted is the mean.

Sympathetic Access. Subjects were tested with respect to their ability to demonstrate effective sympathetic access (Fig. $6)$. In subjects with apparent sympathetic access $(n=7)$, the decay to baseline before and after timolol instillation group means were 29.2 seconds and 69.2 seconds, respectively. In subjects without apparent sympathetic access, the decay to baseline before and after timolol instillation group means were 56.8 seconds and 65.9 seconds, respectively. Two-way, repeated-measures ANOVA for the factors of drug effect and sympathetic access was performed. It revealed a significant effect for drug action $\left(F_{(1,44)}=5.47 ; P=0.02\right)$ but not for sympathetic access $\left(F_{(1,44)}=1.32 ; P=0.25\right)$. Interaction effects were not significant $(P>0.05)$. A post hoc Fisher-LSD test revealed that in subjects with apparent sympathetic access, decay duration after the instillation of timolol was significantly increased $(P=$ 0.02). No other relevant comparisons were significant.

Of the seven subjects exhibiting results suggesting effective sympathetic access, four were emmetropic and three were myopic. Decay durations to baseline before and after timolol

\section{A Sympathetic access}

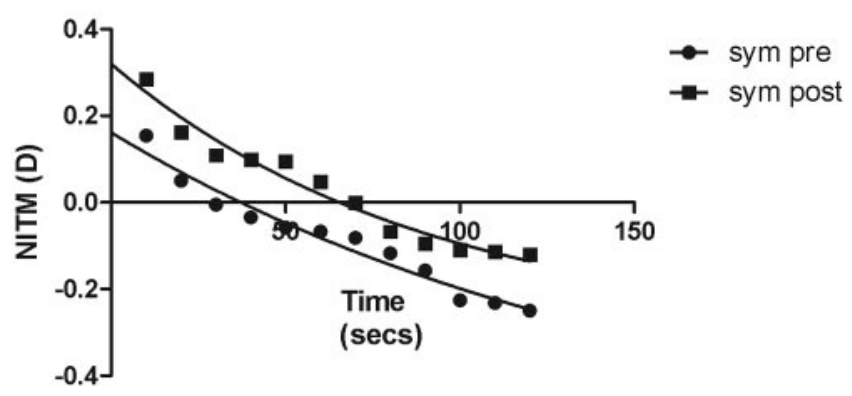

B Without sympathetic access

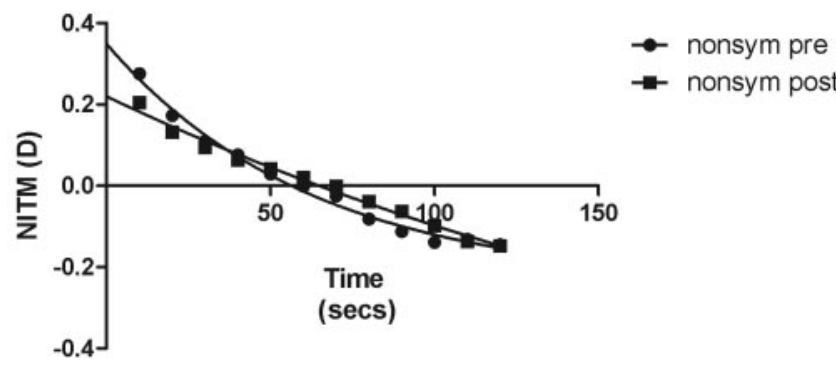

FIGURE 6. Decay of NITM in subjects (top) with sympathetic access and (bottom) without sympathetic access after 1 hour of nearwork before and after instillation of timolol. Plotted is the mean.

\section{Refractive group-Betaxolol}

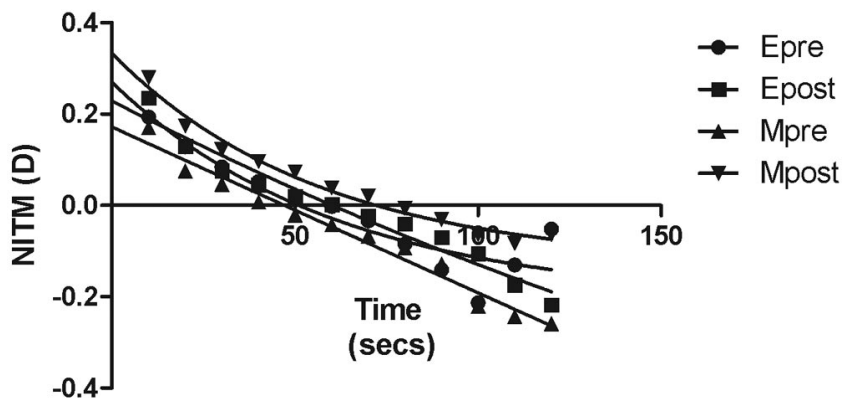

FIGURE 7. Decay of NITM as a function of refractive subgroup after 1 hour of nearwork before and after instillation of betaxolol. Plotted is the mean.

instillation means were 42 seconds and 108 seconds in subjects with myopia and 20 and 43 seconds in subjects with emmetropia, respectively. Paired $t$-tests were performed to compare the NITM decay duration within each of these refractive groups. Only the subjects with myopia demonstrated a significant increase in decay duration with timolol $t_{(2)}=-4.88$; $P=0.03)$; the subjects with emmetropia did $\operatorname{not}\left(t_{(3)}=-2.18\right.$; $P=0.11)$.

Control. Decay duration was assessed in the emmetropic and myopic subgroups (Fig. 7). The decay to baseline before and after betaxolol instillation group means were 52.3 and 72.3 seconds, respectively. In the subjects with emmetropia, the decay to baseline before and after betaxolol instillation group means were 60 and 67.5 seconds, respectively. In the subjects with myopia, the decay to baseline before and after timolol instillation group means were 56.8 and 75 seconds, respectively. Two-way, repeated-measures ANOVA for the factors of drug effect (betaxolol) and refractive group revealed neither a $\operatorname{drug}\left(F_{(1,44)}=0.70 ; P=0.40\right)$ nor a refractive group $\left(F_{(1,44)}=\right.$ $0.02, P=0.88)$ effect. Interaction effects were not significant $(P>0.05)$.

\section{Discussion}

There are two important and new findings in the present study, as assessed under closed-loop viewing conditions. First, with addition of the sympathetic inhibitor timolol, there was a significant increase in NITM decay duration after the sustained 1-hour near task. Furthermore, refractive error susceptibility was found-that is, decay duration was significantly longer in the subjects with myopia. Second, $35 \%(n=7)$ of the subjects demonstrated apparent effective access to sympathetic inhibition under the specific experimental conditions based on the conventional criterion of inherent NITM and decay duration. However, only subjects with myopia $(n=3)$ exhibited a significant effect, representing $15 \%$ of the total sample size.

This is the first study to report on NITM closed-loop accommodative responsivity with and without the pharmacologic addition of timolol. After timolol instillation, the initial NITM magnitude was found to be similar across refractive groups, whereas the decay duration revealed refractive error susceptibility. In other words, the subjects with emmetropia demonstrated a relatively rapid decay back to baseline, whereas the subjects with myopia exhibited a prolonged post-task decay duration. These findings may provide insight into and bear relation to nearwork-induced permanent myopia. ${ }^{10}$ After short durations of nearwork, a small $(\sim 0.3 \mathrm{D})$ and transient myopic shift is typically induced in subjects with myopia. ${ }^{15}$ This myopic shift decays to baseline (in 30 seconds or longer) when one 
performs a low dioptric demand task such as focusing on a distant target, but decay characteristics varied among the refractive groups; they were found to be prolonged in the subjects with myopia. ${ }^{11,16,17}$ Such prolonged decay is consistent with a recently advanced theory of myopia development. ${ }^{24}$ When closely spaced cycles of near-far-near work take place, such as within 30 seconds or less, decay of the NITM may not be complete in some persons. ${ }^{11,25}$ By effectively functioning as a low-powered plus lens, this residual NITM acts to reduce the accommodative stimulus, and in turn the accommodative error ${ }^{26}$ during the subsequent near viewing task, thus promoting emmetropization through axial elongation (for a detailed explanation, see Hung and Ciuffreda ${ }^{24}$ ).

The present findings demonstrated that decay duration in the myopic group was increased after timolol instillation, thus raising an important possible link to myopia development. The sympathetic system may serve in a protective capacity by slightly reducing the accommodative response produced by the parasympathetic innervation during a sustained near activity such as reading. ${ }^{7,15}$ In the present experiment, when timolol was administered, sympathetic innervation was blocked and, thus, its protective function was eliminated. This resulted in increased decay with a potential for myopiagenesis, ${ }^{9-12,20}$ as described earlier.

Previous studies have reported that the accommodative response under open-loop viewing conditions (i.e., in the dark without visual feedback) was modified with the addition of a sympathetic inhibitor, such as timolol. ${ }^{12,20}$ However, they found lack of refractive error dependency. Since the present study was performed using a more naturalistic closed-loop viewing and testing paradigm, and hence included the presence of blur-related visual feedback, it is not surprising that the present results differed from those of previous investigations. In the former, blur feedback had the potential to interact with the pharmacologic effect; in the latter, it did not. This is consistent with the notion that blur processing may be less effective in subjects with myopia. ${ }^{27}$ In addition, these previous studies used short near-task durations (e.g., 3 minutes), but the present investigation incorporated a much longer reading duration (60 minutes).

The present results suggest that subjects exhibiting increased initial NITM with the instillation of timolol have effective access to sympathetic inhibitory facility. A recent study has also provided evidence of such sympathetic inhibitory facility in $27 \%$ of subjects with emmetropia, $21 \%$ of subjects with early-onset myopia, and $29 \%$ of subjects with late-onset myopia (i.e., approximately $25 \%$ of subjects with myopia), and in $26 \%$ of the total group. ${ }^{20}$ Their results are in accord with a previously proposed model related to the role of sympathetic inhibition of accommodation in myopia onset and its progression, which suggested that the sympathetic branch of the autonomic nervous system had reduced function in some subjects. ${ }^{20} \mathrm{~A}$ similar finding was observed in the present study, although it was performed under closed-loop viewing conditions. Based on the initial criteria of the present experiment (see Subjects and Methods), the findings revealed that only 7 of 20 (33\%) subjects demonstrated access to sympathetic inhibition mediated by $\beta_{2}$-adrenoreceptors. These seven subjects exhibited increased initial NITM and a longer decay duration after the 1-hour near task and the subsequent post-task instillation of timolol, whereas the subjects who did not exhibit evidence of a sympathetic inhibitory effect $(n=13)$ did not manifest a significant change in these accommodative response characteristics with timolol. Although all seven subjects (four subjects with emmetropia, three subjects with myopia) demonstrated increased initial NITM and decay duration based on our criteria of any degree of difference, the subjects with myopia demonstrated the largest difference (i.e., between pre- and post- timolol); only that was statistically significant. In contrast, the four subjects with emmetropia did not show a statistically significant difference because the increased NITM and decay duration in them were minimal. Thus, refractive error susceptibility was again suggested. In reality, only 3 of 20 (15\%) subjects exhibited statistically significant sustained and effective sympathetic inhibitory function, which, when pharmacologically blocked, resulted in significantly increased NITM decay duration.

Similar findings of increased NITM decay duration, especially in subjects with myopia, have been reported in several studies that did not involve pharmacologic intervention. ${ }^{11,17,25}$ For example, a recent study on young adults has reported that up to $46 \%$ of the subjects with myopia demonstrated nondecayed residual NITM over a post-task period of 2 minutes. ${ }^{11}$ Similar findings have been reported in children (Ciuffreda and Thunyalukul. IOVS 1999; 40:ARVO E-Abstract 2365; Wolffsohn et al. $^{28}$ ). As described earlier, a proportion of the population that has access to sympathetic inhibitory facility may benefit from its protective function (i.e., it decreases the decay time subsequent to a near task. ${ }^{15}$ Thus, a major finding of the present study-prevalence of substantial sympathetic function in only $15 \%$ of the population-may be considered one of several factors that determine whether a child becomes myopic or remains emmetropic during the early developmental years. This is an important finding that should be tested in young children under naturalistic closed-loop viewing conditions.

What may be the local pharmacologic basis for the nonresponders? It is hypothesized that in nonresponders, the expression of autonomic adrenoreceptor function is attenuated, possibly by differential distribution and density of receptors, ${ }^{6}$ though the pharmacologic/physiologic nature of this attenuation is obscure. When the protective action of the sympathetic function is decreased, such persons may be more susceptible to myopia, as mentioned. It could be hypothesized that to control myopia progression, a selective $\beta_{2}$-agonist (e.g., salbutamol) might be considered. Interestingly, a recent investigation $^{29}$ has examined sympathetic stimulation on accommodation using salbutamol. Chen ${ }^{29}$ reported that this drug attenuated the initial magnitude and decay duration of accommodative adaptation in subjects with progressing myopia. Our results obtained under closed-loop viewing conditions are consistent with the findings of this investigation performed under an open-loop testing paradigm. Thus, the use of salbutamol to control myopia progression should be investigated under closed-loop viewing conditions.

In the present study, timolol modified the accommodative response and produced a decrease in IOP. Measurement of IOP was performed at regular intervals throughout all phases of the test protocol. IOP decreased by an average of $3 \mathrm{~mm} \mathrm{Hg}$ before the near task, and it remained the same after the sustained near task. This reduction in IOP before and after nearwork provides evidence for blocked sympathetic inhibitory function. In contrast, betaxolol produced an average $2.5-\mathrm{mm}$ decrease in IOP only, with no significant change in accommodative response, as expected in its control capacity. This is consistent with the first model involving decreased sympathetic facility alone as an underlying mechanism. ${ }^{12}$

The post-task regression of NITM has been attributed to a physiologic rebound effect. ${ }^{30}$ However, there may be an optical explanation related to the conventional hyperfocal distance refraction. ${ }^{31}$ In this case, the far point of the eye is not made conjugate to infinity; rather, the distal edge of the depth-of-field establishes conjugacy. Because the depth of field dioptrically measures at least $\pm 0.25 \mathrm{D},{ }^{31}$ the far point can be extended distally by $0.25 \mathrm{D}$ or a little more, until true conjugacy with 
infinity is established. The far point may transiently extend to infinity on its dynamic, physiologically based route to reestablishment of the steady state.

The present findings suggest directions for future investigations. First, a longitudinal study involving sympathetic inhibitory function and its effect on myopic growth in children during their rapid ocular growth phase $\left(6-12\right.$ years $\left.^{32}\right)$ is critical. Second, because the prevalence of effective sympathetic inhibitory function was found in a relatively small subset of subjects, future studies should be performed in a larger group of young adults to confirm and extend the finding. Third, investigation of sympathetic inhibitory function in young subjects with progressing myopia could be useful in better understanding the progression of myopia.

\section{References}

1. Ciuffreda KJ. Accommodation, pupil, and presbyopia. In: Benjamin WJ, ed. Borish's Clinical Refraction: Principles and Practice. Philadelphia: WB Saunders; 2006;93-144.

2. Gilmartin B. A review of the role of sympathetic innervation of the ciliary muscle in ocular accommodation. Ophthalmic Physiol Opt. 1986;6:23-37.

3. Culhane HM, Winn B, Gilmartin B. Human dynamic closed-loop accommodation augmented by sympathetic inhibition. Invest Ophthalmol Vis Sci. 1999;40:1137-1143.

4. Tornqvist G. The relative importance of the parasympathetic and sympathetic nervous systems for accommodation in monkeys. Invest Ophthalmol Vis Sci. 1967;6:612-617.

5. Gilmartin B, Bullimore MA, Rosenfield M, Winn B, Owens H. Pharmacological effects on accommodative adaptation. Optom Vis Sci. 1992;69:276-282.

6. Wax MB, Molinoff PB. Distribution and properties of beta-adrenergic receptors in human iris-ciliary body. Invest Ophthalmol Vis Sci. 1987;28:420-430.

7. Gilmartin B, Hogan RE. The role of the sympathetic nervous system in ocular accommodation and ametropia. Ophthalmic Physiol Opt. 1985;5:91-93.

8. Otsuka N, Yoshitomi T, Tsuchiya K, Ukai K, Ishikawa S. Adrenoceptors affect accommodation by modulating cholinergic activity. Jpn J Ophthalmol. 1998;42:66-70.

9. Chen JC, Schmid KL, Brown B. The autonomic control of accommodation and implications for human myopia development: a review. Ophthalmic Physiol Opt. 2003;23:401- 422.

10. Ong E, Ciuffreda KJ. Accommodation, Near work, and Myopia. Santa Ana, CA: Optometric Extension Program Foundation Press; 1997.

11. Vasudevan B, Ciuffreda KJ. Additivity of near work-induced transient myopia (NITM) and its decay characteristics in different refractive groups. Invest Ophthalmol Vis Sci. 2008;49:836-841.

12. Gilmartin B, Winfield NR. The effect of topical beta-adrenoceptor antagonists on accommodation in emmetropia and myopia. Vision Res. 1995;35:1305-1312.
13. Gilmartin B, Bullimore MA. Sustained near-vision augments sympathetic innervation of the ciliary muscle. Clin Vis Sci. 1987;1:197208.

14. Gilmartin B, Bullimore MA. Adaptation of tonic accommodation to sustained visual tasks in emmetropia and late-onset myopia. Optom Vis Sci. 1991;68:22-26.

15. McBrien NA, Millodot M. Differences in adaptation of tonic accommodation with refractive state. Invest Ophthalmol Vis Sci. 1988; 29:460 - 469 .

16. Ciuffreda KJ, Wallis DM. Myopes show increased susceptibility to near work aftereffects. Invest Ophthalmol Vis Sci. 1998;39:17971803.

17. Ciuffreda KJ, Lee M. Differential refractive susceptibility to sustained near work. Ophthalmic Physiol Opt. 2002;22:372-379.

18. Rosenfield M, Gilmartin B. Myopia and Near Work. Boston: Butterworth-Heinemann; 1999

19. Winn B, Culhane HM, Gilmartin B, Strang NC. Effect of betaadrenoceptor antagonists on autonomic control of ciliary smooth muscle. Ophthalmic Physiol Opt. 2002;22:359-365.

20. Mallen EA, Gilmartin B, Wolffsohn JS. Sympathetic innervation of ciliary muscle and oculomotor function in emmetropic and myopic young adults. Vision Res. 2005;45:1641-1651.

21. McBrien NA, Millodot M. Clinical evaluation of the Canon Autoref R-1. Am J Optom Physiol Opt. 1985;62:786-792.

22. Krumholz DM, Fox RS, Ciuffreda KJ. Short-term changes in tonic accommodation. Invest Ophthalmol Vis Sci. 1986;27:552-557.

23. Seidel D, Gray LS, Heron G. The effect of monocular and binocular viewing on the accommodation response to real targets in emmetropia and myopia. Optom Vis Sci. 2005;82:279-285.

24. Hung GK, Ciuffreda KJ. Incremental retinal-defocus theory of myopia development-schematic analysis and computer simulation. Comput Biol Med. 2007;37:930-946.

25. Vera-Diaz FA, Strang NC, Winn B. Near work induced transient myopia during myopia progression. Curr Eye Res. 2002;24:289295.

26. Toates FM. Accommodation function of the human eye. Physiol Rev. 1972;52:828-863.

27. Gwiazda J, Thorn F, Bauer J, Held R. Myopic children show insufficient accommodative response to blur. Invest Ophthalmol Vis Sci. 1993;34:690-694.

28. Wolffsohn JS, Gilmartin B, Li RW, et al. Near work-induced transient myopia in preadolescent Hong Kong Chinese. Invest Ophthalmol Vis Sci. 2003;44:2284-2289.

29. Chen J. Is Autonomic Imbalance a Precursor to Myopia Development (dissertation)? Brisbane, Australia: University of Queensland; 2003.

30. Woung LC, Ukai K, Tsuchiya K, Ishikawa, S. Accommodative adaptation and age of onset of myopia. Ophthal Physiol Opt. 1993; $13: 366-370$

31. Rosenfield M, Ciuffreda KJ, Rosen J. Accommodative response during distance optometric test procedures. J Am Optom Assoc. 1992;63:614-618.

32. Jones LA, Mitchell GL, Mutti DO, Hayes JR, Moeschberger ML, Zadnik K. Comparison of ocular component growth curves among refractive error groups in children. Invest Ophthalmol Vis Sci. $2005 ; 46: 2317-2327$ 